\title{
Postoperative radiotherapy appeared to improve the disease free survival rate of patients with extrahepatic bile duct cancer at high risk of loco-regional recurrence
}

\author{
Mi Young Kim, MD¹, Jin Hee Kim, MD, PhD¹, Yonghoon Kim, MD², Sang Jun Byun, MD³ \\ Departments of ${ }^{1}$ Radiation Oncology and ${ }^{2}$ Surgery, Keimyung University Dongsan Medical Center, \\ Keimyung University School of Medicine, Daegu; ${ }^{3}$ Department of Radiation Oncology, Asan Medical Center, \\ University of Ulsan College of Medicine, Seoul, Korea
}

\begin{abstract}
Purpose: To investigate the outcomes of postoperative radiotherapy (RT), in patients with extrahepatic bile duct (EHBD) cancer by comparing the survival rate between patients undergoing surgery alone or surgery plus postoperative RT, and to identify the prognostic factors affecting survival.

Materials and Methods: Between 2000 and 2013, 52 patients with EHBD cancer underwent surgical resection. Of these, 33 patients did not receive postoperative RT (group I), and 19 patients did (group II). R1 resection was significantly more frequent in group II. The median radiation dose was 5,040 cGy.

Results: The 3-year overall survival (OS) rate for group I and group II was 38\% and $56 \%$, respectively ( $p=0.274)$. The 3 -year disease free survival (DFS) rate for group I and group II was $20 \%$ and $31 \%$, respectively $(p=0.049)$, and the 3 -year loco-regional recurrence free survival (LRFS) rates were 19\% and 58\%, respectively $(p=0.002)$. Multivariate analyses showed that postoperative RT and lymphovascular invasion were independent prognostic factors for DFS and LRFS. Overall, 42 patients (80\%) experienced treatment failure. Distant metastasis was the predominant pattern of failure in group II.

Conclusion: Postoperative RT after surgical resection appeared to improve the loco-regional control and DFS rate. More effort is needed to reduce distant metastasis, the major pattern of failure, in patients who receive postoperative RT.
\end{abstract}

Keywords: Extrahepatic bile duct cancer, Postoperative, Radiotherapy, Survival

\section{Introduction}

According to the cancer registries in Korea, extrahepatic bile duct (EHBD) cancer is a rare malignancy [1]. In patients with resectable EHBD cancer, complete surgical resection is the only treatment with curative potential. However, complete surgical resection with a negative resection margin is difficult, because of the critical adjacent structures, such as the hepatic artery and the main portal vein. Even after complete surgical resection, the major pattern of failures is loco-regional recurrence $[2,3]$ and the overall survival (OS) rate of patients who undergo complete resection was only 10\%-40\% [2-8]. Considering that the major pattern of failure is loco-regional recurrence $[3,4,7]$, many investigators have studied the role of

Received 19 July 2016, Revised 26 August 2016, Accepted 4 October 2016.

Correspondence: Jin Hee Kim, MD, PhD, Department of Radiation Oncology, Keimyung University Dongsan Medical Center, Keimyung University School of Medicine, 56 Dalseong-ro, Jung-gu, Daegu 41931, Korea. Tel: +82-53-250-7665, Fax: +82-53250-7487, E-mail: jhkim@dsmc.or.kr

(C) This is an Open Access article distributed under the terms of the Creative Commons Attribution Non-Commercial License (http://creativecommons.org/ licenses/by-nc/4.0/) which permits unrestricted non-commercial use, distribution, and reproduction in any medium, provided the original work is properly cited.

www.e-roj.org 
postoperative radiotherapy (RT) with or without chemotherapy. However, because of the rarity of EHBD cancer, no randomized studies have investigated the benefits of postoperative RT. Based on the results of retrospective studies, many institutions recommend postoperative RT for patients with microscopic/ macroscopic residual tumor or positive regional lymph nodes. However, the clinical benefits of RT are still a debated.

This study was performed to investigate the outcomes of postoperative RT, in patients with EHBD cancer by comparing the survival rates of patients who underwent surgery alone or surgery plus postoperative RT, and to identify the prognostic factors affecting survival in these patients.

\section{Materials and Methods}

\section{Patient selection}

Seventy patients were histologically diagnosed with EHBD cancer between January 2000 and December 2013 at our institution. Of these, 52 with non-metastatic EHBD cancer underwent surgical resection and were analyzed in this study. The other 18 patients were excluded because they had an unresectable tumor $(n=17)$, or distant metastasis $(n=$ 1). After obtaining Institutional Review Board approval, the medical records of 52 patients retrieved and analyzed.

\section{Treatment}

For proximal tumors, liver lobectomy with bile duct resection or bile duct resection with hepaticojejunostomy was conducted with a curative aim. For distal tumors, the Whipple procedure (pancreaticoduodenectomy), pylorus-preserving pancreaticoduodenectomy (PPPD) or bile duct resection was conducted. Regional lymph node dissection was performed.

Postoperative RT is generally recommended for patients with R1 or R2 (macroscopically residual tumor) resection or regional lymph node metastasis. RT was generally started $4-8$ weeks after surgery. All patients underwent three-dimensional conformal RT. The clinical target volume (CTV) was defined as the tumor bed and the regional lymph node area. The planning target volume (PTV) included the CTV and an additional margin, considering setup error and breathing motion. External beam RT was delivered with multiple fields using $15 \mathrm{MV}$ photon beams. The median radiation dose was 5,040 cGy (range, 4,500 to 5,400 cGy).

\section{Statistical analysis}

The independent samples t-test or Pearson $\chi^{2}$ test were used to compare variables between the two groups. All events were calculated from the date of surgery to the date of the event or the last follow-up visit. Loco-regional failure was defined as any recurrence in the primary tumor bed or regional lymphatics. Distant metastasis was defined as any recurrence in a systemic organ. The OS, disease free survival (DFS) and loco-regional recurrence free survival (LRFS) were calculated using the Kaplan-Meier method. The Cox proportional hazards model was used to identify the prognostic factors for survival in multivariate analysis. Statistical analyses were carried out using SPSS ver. 21.0 (IBM, Armonk, NY, USA)

\section{Results}

\section{Patient's and treatment characteristics}

The characteristics of patients are summarized in Table 1. Thirty three patients did not receive postoperative RT (group I), and the remaining 19 patients received postoperative RT (group II). The median age was 65 years (range, 43 to 81 years), and was similar in both groups. The tumor was located in the proximal region in 22 patients, and in the distal region in 30 patients. Proximal tumor was defined anatomically as tumors located in the extrahepatic biliary duct proximal to the origin of the cystic duct. Distal tumor was located in the biliary duct distal to the origin of the cystic duct. Tumor stage was determined according to the American Joint Committee on Cancer staging system (7th edition). There were no significant differences in the tumor stage, surgical procedures, and pathologic characteristics, such as differentiation, perineural invasion, and lymphovascular invasion, between the two groups.

After surgical resection, Ro resection (pathologically negative margin) was achieved in 28 patients (23 patients in group I and 5 patients in group II). The proportion of patients with $\mathrm{R} 1$ resection (microscopically positive margin) was significantly greater in group II than group I. All 5 patients with RO resection in group II had positive lymph node involvement or high grade dysplasia at resection margin. The 10 patients with $\mathrm{R} 1$ resection in group I did not receive postoperative RT at the surgeon's discretion. During RT, 12 patients received concurrent chemotherapy. Eight patients received 5-fluorouracil plus cisplatin; 2 patients received 5 -fluorouracil and leucovorin; and the other 2 patients received weekly gemcitabine. No patients received maintenance chemotherapy after concurrent chemoradiotherapy (CCRT). No patients in group I received chemotherapy as adjuvant setting.

\section{Survival and prognostic factors}

The median follow-up duration in all patients was 24 months 
Table 1. Patients and tumor characteristics

\begin{tabular}{|c|c|c|c|}
\hline Variable & $\begin{array}{l}\text { Group I } \\
(n=33)\end{array}$ & $\begin{array}{l}\text { Group II } \\
(n=19)\end{array}$ & $p$-value ${ }^{a)}$ \\
\hline Age (yr) & $65(43-79)$ & $67(46-81)$ & 0.465 \\
\hline Gender & & & 0.760 \\
\hline Male & $23(70)$ & $14(74)$ & \\
\hline Female & $10(30)$ & $5(26)$ & \\
\hline Tumor site & & & 0.077 \\
\hline Proximal & 17 (52) & $5(26)$ & \\
\hline Distal & $16(48)$ & $14(74)$ & \\
\hline T stage $e^{b)}$ & & & 0.427 \\
\hline $\mathrm{T} 1$ & $7(22)$ & $3(16)$ & \\
\hline $\mathrm{T} 2$ & $16(48)$ & $8(42)$ & \\
\hline T3 & $10(30)$ & $8(42)$ & \\
\hline$N$ stage $^{b)}$ & & & 0.272 \\
\hline No & $24(73)$ & $11(58)$ & \\
\hline N1 & $9(27)$ & $8(42)$ & \\
\hline Stage $e^{b)}$ & & & 0.781 \\
\hline I & $10(30)$ & $7(37)$ & \\
\hline ॥ & $20(60)$ & $11(58)$ & \\
\hline III & $3(10)$ & $1(5)$ & \\
\hline Surgical procedure & & & 0.224 \\
\hline PPPD & $8(24)$ & $9(47)$ & \\
\hline Whipple procedure & $2(6)$ & $0(0)$ & \\
\hline Bile duct resection & $10(30)$ & $6(32)$ & \\
\hline $\begin{array}{l}\text { Liver lobectomy with } \\
\text { bile duct resection }\end{array}$ & $13(40)$ & $4(21)$ & \\
\hline Residual tumor & & & 0.004 \\
\hline R0 resection & $23(70)$ & $5(26)$ & \\
\hline $\mathrm{R} 1$ resection & $10(30)$ & $14(74)$ & \\
\hline Differentiation & & & 0.747 \\
\hline Well & $9(27)$ & $3(16)$ & \\
\hline Moderate & 17 (52) & $10(53)$ & \\
\hline Poor & $6(18)$ & $5(26)$ & \\
\hline Unknown & $1(3)$ & $1(5)$ & \\
\hline Perineural invasion & & & 0.662 \\
\hline Yes & $23(70)$ & $12(63)$ & \\
\hline No & $7(21)$ & $6(32)$ & \\
\hline Unknown & $3(9)$ & $1(5)$ & \\
\hline Lymphovascular invasion & & & 0.765 \\
\hline Yes & $19(58)$ & $10(53)$ & \\
\hline No & $11(32)$ & $8(42)$ & \\
\hline Unknown & $3(10)$ & $1(5)$ & \\
\hline
\end{tabular}

Values are presented as median (range) or number (\%).

PPPD, pylorus-preserving pancreaticoduodenectomy; RO, negative resection margin; $R 1$, microscopically positive margin.

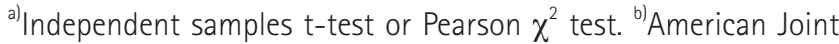
Committee on Cancer staging system (7th edition).

(range, 3 to 105 months). The median OS for group I and group II was 26 months and 37 months, respectively. The 3-year OS rates for group I and group II were $38 \%$ and $56 \%$, respectively,

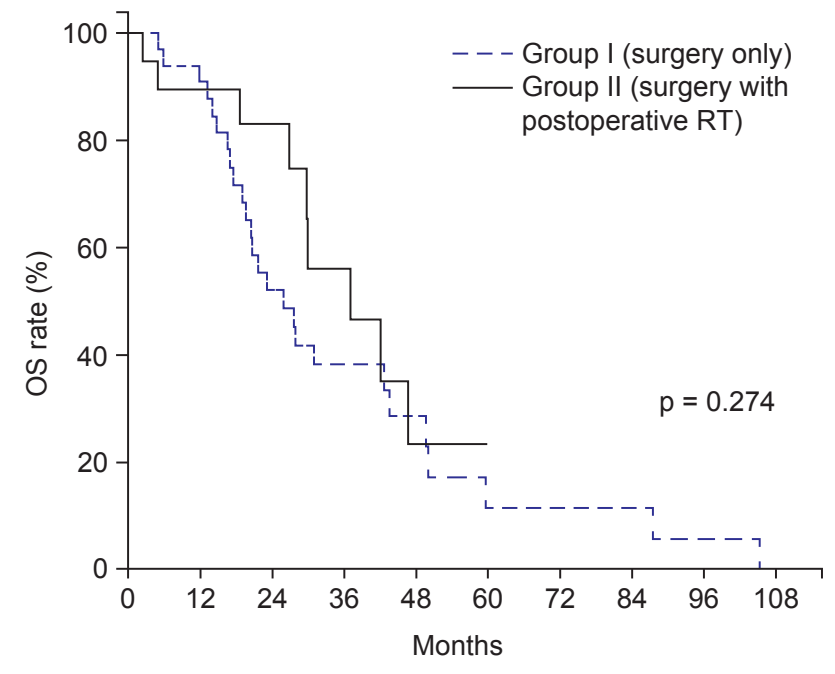

Fig. 1. Overall survival (OS) curve. The 3-year OS rates for group I and group II were $38 \%$ and $56 \%$, respectively. RT, radiotherapy.

and they were not significantly different between the two groups ( $p=0.274)$ (Fig. 1). The 3-year DFS rates for Group I and Group II were 20\% and 31\%, respectively $(p=0.049)$ (Fig. $2 A)$, and the 3 -year LRFS were $19 \%$ and $58 \%$, respectively ( $p=$ 0.002) (Fig. 2B).

In univariate analyses of the prognostic factors for OS (Table 2), the advanced T stage, $N$ stage, perineural invasion, and lymphovascular invasion were significantly associated with poor prognosis. Postoperative RT, and lymphovascular invasion were significant prognostic factors for DFS. RO resection, no postoperative RT, no use of chemotherapy, perineural invasion, and lymphovascular invasion were significant prognostic factors for lower 3-year LRFS.

Variables with a $p$-value $<0.2$ on univariate analysis were incorporated into multivariate analysis. However, chemotherapy was excluded, because all patients received chemotherapy were in group II. Result showed that postoperative RT and Iymphovascular invasion were independent prognostic factors for DFS. Lymphovascular invasion was the only independent prognostic factor for OS (Table 3).

To determine the survival benefit of postoperative RT in patients with $R 1$ resection, we did subgroup analysis: $R 1$ resection with $R T(n=14), R 1$ resection without $R T(n=10)$, $R 0$ resection with $R T(n=5)$, R0 resection without $R T$ ( $n=$ 23). The 3-year DFS rates were $28 \%, 33 \%, 40 \%$, and $14 \%$, respectively. The 3 -year LRFS rates were $48 \%, 30 \%, 60 \%$, and $14 \%$, respectively. There were significant difference of the 3-year DFS and LRFS between R1 resection with RT and R0 
A

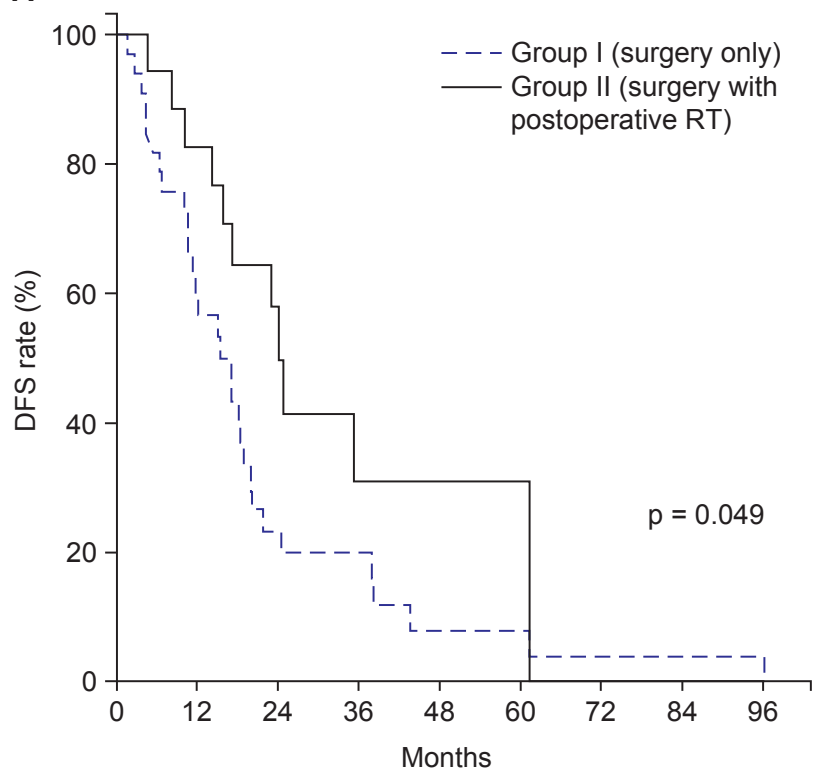

B

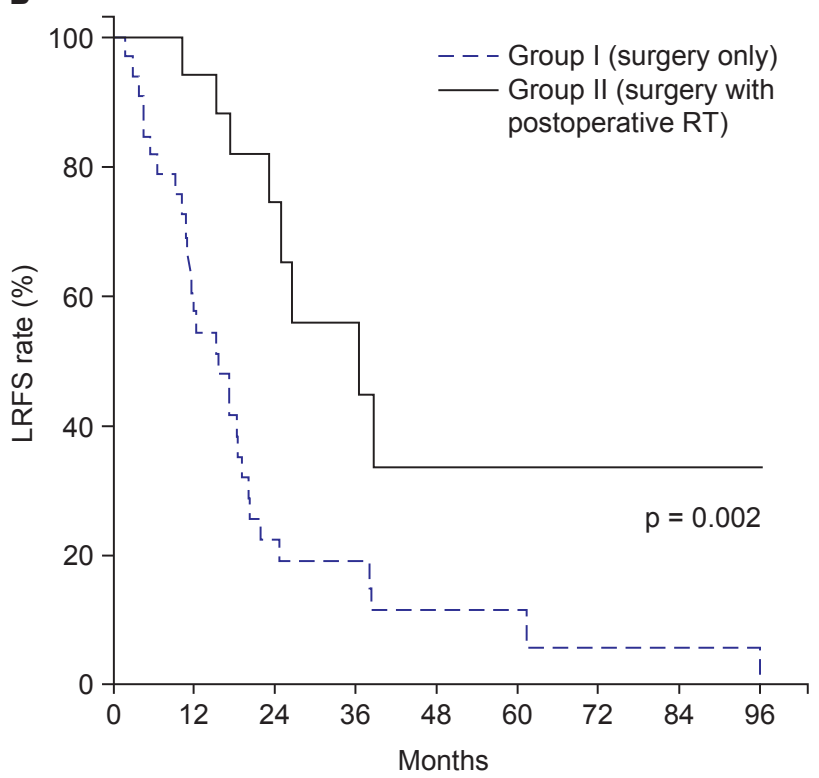

Fig. 2. (A) Disease-free survival (DFS) curve and (B) locoregional recurrence-free survival (LRFS) curve. The 3-year DFS rates for group I and group II were 20\% and 31\%, respectively, and the 3 -year LRFS rates were $19 \%$ and $58 \%$, respectively. RT, radiotherapy.

Table 2. Univariate analysis of overall survival (OS), disease-free survival (DFS), and locoregional recurrence-free survival (LRFS)

\begin{tabular}{|c|c|c|c|c|c|c|c|}
\hline \multirow{2}{*}{ Variable } & \multirow{2}{*}{ No. of patients } & \multicolumn{6}{|c|}{ 3-yr survival rate (\%) } \\
\hline & & OS & $p$-value & DFS & $p$-value & LRFS & $p$-value \\
\hline Age (yr) & & & 0.335 & & 0.077 & & 0.068 \\
\hline$\leq 60$ & 13 & 42 & & 12 & & 23 & \\
\hline$>60$ & 39 & 46 & & 29 & & 24 & \\
\hline T stage & & & 0.040 & & 0.199 & & 0.076 \\
\hline $\mathrm{T} 1-2$ & 34 & 52 & & 27 & & 34 & \\
\hline T3 & 18 & 29 & & 21 & & 17 & \\
\hline N stage & & & 0.025 & & 0.071 & & 0.126 \\
\hline No & 35 & 56 & & 31 & & 37 & \\
\hline N1 & 17 & 22 & & 14 & & 20 & \\
\hline Residual disease & & & 0.482 & & 0.171 & & 0.039 \\
\hline RO & 28 & 43 & & 19 & & 23 & \\
\hline R1 & 24 & 46 & & 34 & & 44 & \\
\hline Radiotherapy & & & 0.274 & & 0.049 & & 0.002 \\
\hline Yes & 19 & 56 & & 31 & & 58 & \\
\hline No & 33 & 38 & & 20 & & 19 & \\
\hline Chemotherapy & & & 0.203 & & 0.226 & & 0.045 \\
\hline Yes & 12 & 53 & & 26 & & 53 & \\
\hline No & 40 & 42 & & 24 & & 25 & \\
\hline Perineural invasion & & & 0.037 & & 0.069 & & 0.020 \\
\hline Yes & 35 & 41 & & 22 & & 26 & \\
\hline No & 17 & 70 & & 39 & & 56 & \\
\hline Lymphovascular invasion & & & 0.015 & & 0.013 & & 0.005 \\
\hline Yes & 29 & 39 & & 11 & & 16 & \\
\hline No & 23 & 65 & & 49 & & 60 & \\
\hline
\end{tabular}

$\mathrm{RO}$, negative resection margin; $\mathrm{R} 1$, microscopically positive margin. 
Table 3. Multivariate analysis of prognostic factors for overall survival (OS), disease-free survival (DFS), locoregional recurrence-free survival (LRFS)

\begin{tabular}{|c|c|c|c|c|c|c|}
\hline \multirow{2}{*}{ Variable } & \multicolumn{2}{|l|}{ OS } & \multicolumn{2}{|l|}{ DFS } & \multicolumn{2}{|l|}{ LRFS } \\
\hline & $\operatorname{HR}(95 \% \mathrm{Cl})$ & $p$-value & $\mathrm{HR}(95 \% \mathrm{Cl})$ & p-value & $\mathrm{HR}(95 \% \mathrm{Cl})$ & $p$-value \\
\hline T stage & - & 0.152 & - & 0.311 & - & 0.202 \\
\hline N stage & - & 0.226 & - & 0.376 & - & 0.665 \\
\hline Residual disease & - & 0.935 & - & 0.904 & - & 0.808 \\
\hline Postoperative radiotherapy & - & 0.324 & $0.484(0.238-0.985)$ & $0.045^{*}$ & $0.287(0.128-0.642)$ & $0.002^{*}$ \\
\hline Perineural invasion & - & 0.350 & - & 0.464 & - & 0.296 \\
\hline Lymphovascular invasion & $2.271(1.261-4.091)$ & $0.006^{*}$ & 2.100 (1.264-3.489) & $0.004^{*}$ & $2.494(1.444-4.307)$ & $0.001^{*}$ \\
\hline
\end{tabular}

$\mathrm{HR}$, hazard ratio; $\mathrm{Cl}$, confidence interval.

*Statistically significant at $p<0.05$.

A

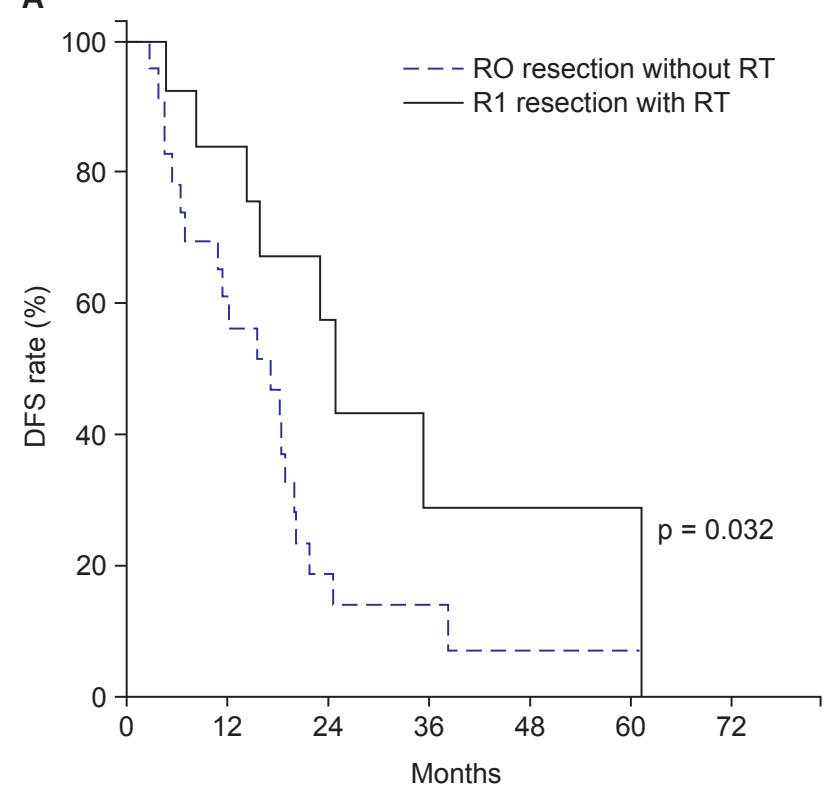

B

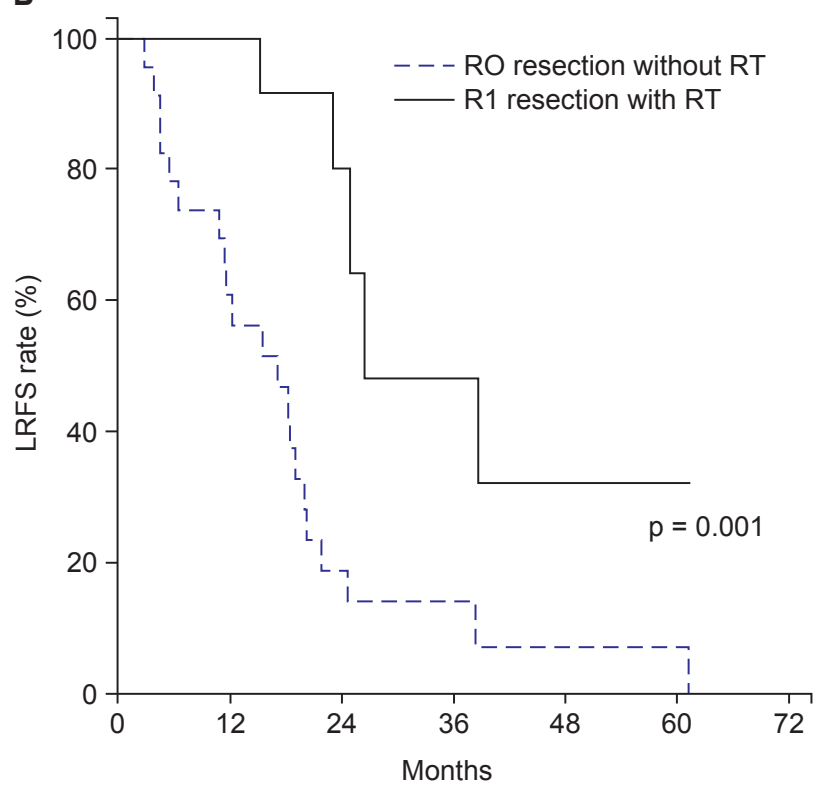

Fig. 3. (A) Disease-free survival (DFS) curve and (B) locoregional recurrence-free survival (LRFS) curve for patients with R1 resection with RT vs. patients with $\mathrm{R} 0$ resection without $\mathrm{RT}$. R0, negative resection margin; $\mathrm{R} 1$, microscopically positive margin; $R T$, radiotherapy.

resection without RT ( $p=0.032$ for DFS, $p=0.001$ for LRFS) (Fig. 3), and age, gender, tumor location, stage, perineural invasion, and lymphovascular invasion were not significantly different between these two groups. However, the OS rate was not significantly different between these two groups $(p=0.762)$.

\section{Patterns of failure}

A total of 42 patients (80\%) experienced treatment failure (Table 4). For group I, 26 patients experienced locoregional failure at median 14 months after surgery (range, 2 to 24 months). For group I, there was a statistically significant increase in overall loco-regional failure rate compared with group II (84\% and 21\% for group I and group II, respectively;
Table 4. Patterns of treatment failure

\begin{tabular}{lcc}
\hline \multicolumn{1}{c}{ Site of recurrence } & Group I $(n=33)$ & Group II $(n=19)$ \\
\hline Locoregional & 26 & 1 \\
Distant & 3 & 7 \\
Locoregional + distant & 2 & 3 \\
Total & $31(93 \%)$ & $11(58 \%)$ \\
\hline
\end{tabular}

$p$-value $=0.001)$. For group II patient, distant metastasis was predominant pattern of failure.

Of 26 patients with locoregional recurrence in group I, 23 patients received salvage treatment, including CCRT in 15 patients, RT alone 7 patients, and chemotherapy alone in 1 
patients. Median radiation dose was 5,040 cGy (range, 4,500 to 5,400 cGy). After salvage treatment, the median survival time was 7.5 months and the 1 -year survival rate was $33 \%$. One patient with locoregional recurrence in group $\mathrm{I}$, he could not receive any salvage treatment due to poor performance status.

\section{Toxicity of adjuvant treatment}

Treatment-related toxicity was evaluated using the Common Terminology Criteria for Adverse Events (version 4.0). In most cases, adjuvant treatment was well tolerated. Fourteen patients experienced grade 1 gastrointestinal complication, including abdominal pain, and anorexia. Two patients experienced grade 3 duodenal ulcer at 14 and 36 months after RT. These 2 patients received radiation doses of 5,400 and 5,040 $c G y$, respectively. Both patients recovered after endoscopic coagulation treatment. There were no treatment related deaths.

\section{Discussion and Conclusion}

The high incidence of loco-regional recurrence after surgical resection has provided the rationale for postoperative RT. Jarnagin et al. [3], reported the patterns of initial disease recurrence after resection of hilar cholangiocarcinoma. At a median follow-up of 24 months, 52 of 76 patients developed tumor recurrence, which occurred loco-regionally in 65\% of these patients. Similar results were reported by Koo et al. [4], who analyzed 97 patients with resected EHBD cancer. The location of the tumor was classified as proximal in 26 patients, and distal in 71 patients. Loco-regional failure was the most common pattern of initial failure. Treatment failure was locoregional in $79 \%$ and $81 \%$ of patients with proximal and distal DHBD cancer, respectively. In the present study, a total of 42 patients (80\%) experienced treatment failure. In patients who underwent surgery alone (group I), the most common pattern of failure was loco-regional recurrence.

Based on these results, postoperative RT might be effective in terms of improving loco-regional tumor control, and hence increase survival. A comparative study by Gwak et al. [7] reported that postoperative RT decreased the local failure rate (61.7\% for surgery alone compared with 35.6\% for surgery plus postoperative RT). Among patients with R1 resection, postoperative RT increased the DFS rate. In the study by Borghero et al. [9], the OS and loco-regional recurrence outcomes for patients with standard-risk Ro resection and negative lymph node involvement were compared to those of patients with high loco-regional recurrence risk with R1 resection and/or positive lymph node status who received postoperative chemoradiotherapy. They reported that there were no differences in the survival and loco-regional recurrence rates between these two groups. These findings suggested that postoperative chemoradiation provided an

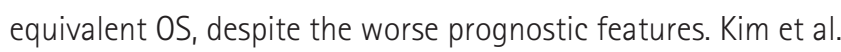
[10] reported that postoperative chemoradiation could improve long term survival, especially for patients with loco-regional control. The 5 -year OS rate was $44.7 \%$ in those patients. In the present study, the DFS and LRFS rates were higher in patients who received postoperative $\mathrm{RT}$, indicating that postoperative $\mathrm{RT}$ reduced the loco-regional failure rate.

Various factors were reported to be associated with the outcomes of EHBD cancer. In particular, several studies showed that the resection margin status, T stage, N stage, perineural invasion, poor histologic differentiation, or postoperative carbohydrate antigen 19-9 concentration were prognostic factors $[4,11-14]$. The resection margin status is a wellestablished prognostic factor for loco-regional control and/or OS. After postoperative RT, however, some studies showed that there was no difference in OS between patients with a negative or positive resection margin cases $[15,16]$. These findings suggest that postoperative RT could be beneficial for patients with a positive resection margin after surgical resection. Horgan et al. [17] conducted a meta-analysis to determine the impact of adjuvant therapy for biliary tract cancers including gallbladder cancer. For patients with $\mathrm{R} 1$ resection, there was a benefit of adjuvant $\mathrm{RT}$ (odds ratio [OR], 0.33; $p=0.01$ ). In the present study, we compared the survival rates between patients with $\mathrm{R} 1$ resection who received postoperative $\mathrm{RT}$ and patients with $\mathrm{R} 0$ resection without postoperative $\mathrm{RT}$ to clarify the role of postoperative RT for patients with $\mathrm{R} 1$ resection. The 3-year DFS and LRFS rates were higher in patients with $\mathrm{R} 1$ resection who received postoperative $\mathrm{RT}$ than those for patients with $\mathrm{RO}$ resection who did not receive postoperative $\mathrm{RT}$, although the $\mathrm{R} 1$ resection is associated with poor locoregional control. These findings supported that postoperative RT had beneficial effects in EHBD cancer patients with R1 resection who underwent surgical resection.

In univariate analysis, the T stage was associated with OS, but not with DFS or LRFS, in this study. When patients were divided by $T$ stage, there were no differences in the patterns of failure and rates of salvage treatment (data not shown). After salvage treatment, the 1-year OS rates after salvage treatment were $40 \%$ and $22 \%$, for T1-2 and T3 patients, respectively (although, no statistically significant). This might explain the difference in OS according to T stage. 
In the present study, the predominant pattern of failure was distant metastasis in patients who received postoperative RT, primarily as a result of increased loco-regional control. Similarly, many retrospective studies have suggested that postoperative RT improved local control and survival, although distant metastasis was the most common pattern of failure $[7,9,10,18,19]$. In the meta-analysis by Horgan et al. [17], adjuvant treatment after surgery for bile duct cancer improved survival compared with surgery alone (although this was not statistically significant; $O R, 0.74 ; p=0.06$ ). In a sensitivity analysis, adjuvant chemotherapy or CCRT were associated with greater benefit than RT alone $(0 R, 0.39,0.61$, and 0.98 , respectively; $p=0.02$ ). Given that major pattern of failure who received adjuvant radiotherapy was distant metastasis, the study is needed to determine the benefit of addition chemotherapy to RT or maintenance chemotherapy after CCRT. Recently, a single-arm, phase II SWOG study examined the efficacy of adjuvant chemotherapy and chemoradiotherapy in patients with resected EHBD cancer or gallbladder cancer [20]. The eligibility criteria were patients with having stage pT2-4 or $\mathrm{N}(+)$ or a positive resection margin. Patients received four cycles of gemcitabine and capecitabine followed by capecitabine with RT. This treatment regimen was well tolerated and promising efficacy with the 2-year OS rate of $65 \%$ and the median 05 of 35 months. The National Comprehensive Cancer Network guidelines recommend fluoropyrimidine based chemoradiation followed by additional fluoropyrimidine-based or gemcitabine-based chemotherapy for patients with a positive resection margin or positive regional lymph nodes after curative resection [21].

This study had some limitations, including its retrospective, nonrandomized design, and the relatively small number of patients who received postoperative RT. Because this study was not performed prospectively, the characteristics of patients in both groups were not well matched. $\mathrm{R} 1$ resection was more frequent in the group II than in group I. The group I comprised a greater number of patients than the group II (33 patients for group I, and 19 patients for group II). However, the DFS and LRFS rates were greater in the group I and the OS rate was not significantly different between the two groups; even though $\mathrm{R} 1$ resection was more frequent in the group II. This finding could provide further insights into the treatment strategy for EHBD cancer after curative surgery.

In conclusions, postoperative RT after surgical resection appeared to improve the loco-regional control and DFS rates, especially in patients with $\mathrm{R} 1$ resection. More effort is needed to reduce distant metastasis, which is the major pattern of failure in patients who received postoperative RT. Intensification of systemic chemotherapy may be necessary in such patients. In addition, a prospective randomized trial is needed to confirm our results.

\section{Conflict of Interest}

No potential conflict of interest relevant to this article was reported.

\section{References}

1. Jung KW, Won YJ, Kong HJ, et al. Cancer statistics in Korea: incidence, mortality, survival, and prevalence in 2012. Cancer Res Treat 2015;47:127-41.

2. Kopelson G, Galdabini J, Warshaw AL, Gunderson LL. Patterns of failure after curative surgery for extra-hepatic biliary tract carcinoma: implications for adjuvant therapy. Int J Radiat Oncol Biol Phys 1981;7:413-7.

3. Jarnagin $W R$, Ruo $L$, Little $S A$, et al. Patterns of initial disease recurrence after resection of gallbladder carcinoma and hilar cholangiocarcinoma: implications for adjuvant therapeutic strategies. Cancer 2003;98:1689-700.

4. Koo TR, Eom KY, Kim IA, et al. Patterns of failure and prognostic factors in resected extrahepatic bile duct cancer: implication for adjuvant radiotherapy. Radiat Oncol J 2014;32:63-9.

5. Gazzaniga GM, Filauro M, Bagarolo C, Mori L. Surgery for hilar cholangiocarcinoma: an Italian experience. J Hepatobiliary Pancreat Surg 2000;7:122-7.

6. Nakeeb A, Pitt HA, Sohn TA, et al. Cholangiocarcinoma. A spectrum of intrahepatic, perihilar, and distal tumors. Ann Surg 1996;224:463-73.

7. Gwak HK, Kim WC, Kim HJ, Park JH. Extrahepatic bile duct cancers: surgery alone versus surgery plus postoperative radiation therapy. Int J Radiat Oncol Biol Phys 2010;78:194-8.

8. Jang JY, Kim SW, Park DJ, et al. Actual long-term outcome of extrahepatic bile duct cancer after surgical resection. Ann Surg 2005;241:77-84.

9. Borghero Y, Crane CH, Szklaruk J, et al. Extrahepatic bile duct adenocarcinoma: patients at high-risk for local recurrence treated with surgery and adjuvant chemoradiation have an equivalent overall survival to patients with standard-risk treated with surgery alone. Ann Surg Oncol 2008;15:3147-56.

10. Kim K, Chie EK, Jang JY, et al. Adjuvant chemoradiotherapy after curative resection for extrahepatic bile duct cancer: a long-term single center experience. Am J Clin Oncol 2012;35:136-40.

11. Lillemoe KD, Cameron JL. Surgery for hilar cholangiocarcinoma: the Johns Hopkins approach. J Hepatobiliary Pancreat Surg 
2000;7:115-21.

12. Jarnagin $W R$, Fong $Y$, DeMatteo $R P$, et al. Staging, resectability, and outcome in 225 patients with hilar cholangiocarcinoma. Ann Surg 2001;234:507-17.

13. Nakeeb A, Tran KO, Black MJ, et al. Improved survival in resected biliary malignancies. Surgery 2002;132:555-63.

14. Park JH, Choi EK, Ahn SD, et al. Postoperative chemoradiotherapy for extrahepatic bile duct cancer. Int J Radiat Oncol Biol Phys 2011;79:696-704.

15. Kim S, Kim SW, Bang YJ, Heo DS, Ha SW. Role of postoperative radiotherapy in the management of extrahepatic bile duct cancer. Int J Radiat Oncol Biol Phys 2002;54:414-9.

16. Stein DE, Heron DE, Rosato EL, Anne PR, Topham AK. Positive microscopic margins alter outcome in lymph node-negative cholangiocarcinoma when resection is combined with adjuvant radiotherapy. Am J Clin Oncol 2005;28:21-3.

17. Horgan AM, Amir E, Walter T, Knox JJ. Adjuvant therapy in the treatment of biliary tract cancer: a systematic review and meta-analysis. J Clin Oncol 2012;30:1934-40.

18. Matsuda $T$, Fujita $H$, Harada $N$, et al. Impact of adjuvant radiation therapy for microscopic residual tumor after resection of extrahepatic bile duct cancer. Am J Clin Oncol 2013;36:461-5.

19. Nelson JW, Ghafoori AP, Willett CG, et al. Concurrent chemoradiotherapy in resected extrahepatic cholangiocarcinoma. Int J Radiat Oncol Biol Phys 2009;73:148-53.

20. Ben-Josef E, Guthrie KA, El-Khoueiry AB, et al. SWOG S0809: a phase II intergroup trial of adjuvant capecitabine and gemcitabine followed by radiotherapy and concurrent capecitabine in extrahepatic cholangiocarcinoma and gallbladder carcinoma. J Clin Oncol 2015;33:2617-22.

21. National Comprehensive Cancer Network. Hepatobiliary cancer (version 2. 2016) [Internet]. Fort Washington, PA: National Comprehensive Cancer Network; c2016 [cited 2016 Oct 24]. Available from: https://www.nccn.org/professionals/physician_ gls/pdf/hepatobiliary.pdf. 